\title{
The mechanism and detection of alternative splicing events in circular RNAs
}

\author{
Xiaohan Li ${ }^{1}$, Bing Zhang ${ }^{1}$, Fuyu Li $^{1}{ }^{1}$ Kequan Yu ${ }^{1}$, Yunfei Bai ${ }^{\text {Corresp. } 1}$ \\ ${ }^{1}$ State Key Laboratory of Bioelectronics, School of Biological Sciences and Medical Engineering, Southeast University, Nanjing, Jiangsu, China \\ Corresponding Author: Yunfei Bai \\ Email address: whitecf@seu.edu.cn
}

Circular RNAs (circRNAs) are considered as functional biomolecules with tissue/development-specific expression patterns. Generally, a single gene may generate multiple circRNA variants by alternative splicing, which contain different combinations of exons and/or introns. Due to the low abundance of circRNAs as well as overlapped with their linear counterparts, circRNA enrichment protocol is needed prior to sequencing. Compared with numerous algorithms, which use back-splicing reads for detection and functional characterization of circRNAs, original bioinformatic analyzing tools have been developed to large-scale determination of full-length circRNAs and accurate quantification. This review provides insights into the complexity of circRNA biogenesis and surveys the recent progresses in the experimental and bioinformatic methodologies that focus on accurately full-length circRNAs identification. 
1 The mechanism and detection of alternative splicing

2 events in circular RNAs

3

4

5

6

7

8

9

10

11

12

13

14

15

16

17

18

19

20

21

22

23

24

25

26

27

28

29

30

31

32

33

34

35

36

37

38

39

Xiaohan Li ${ }^{1}$, Bing Zhang ${ }^{1}$, Fuyu Li ${ }^{1}$, Kequan Yu ${ }^{1}$, Yunfei Bai Corresp. 1

${ }^{1}$ State Key Lab of Bioelectronics, School of Biological Science and Medical Engineering, Southeast University, Nanjing, Jiangsu, 210096, China

Corresponding Author:

Yunfei Bai

Southeast University, Sipailou No.2, Nanjing, Jiangsu 210096, China

Email address: whitecf@seu.edu.cn

\section{Abstract}

Circular RNAs (circRNAs) are considered as functional biomolecules with tissue/developmentspecific expression patterns. Generally, a single gene may generate multiple circRNA variants by alternative splicing, which contain different combinations of exons and/or introns. Due to the low abundance of circRNAs as well as overlapped with their linear counterparts, circRNA enrichment protocol is needed prior to sequencing. Compared with numerous algorithms, which use back-splicing reads for detection and functional characterization of circRNAs, original bioinformatic analyzing tools have been developed to large-scale determination of full-length circRNAs and accurate quantification. This review provides insights into the complexity of circRNA biogenesis and surveys the recent progresses in the experimental and bioinformatic methodologies that focus on accurately full-length circRNAs identification.

\section{Introduction}

Circular RNAs (circRNAs), without 5'-3' polarity and polyadenylated (poly(A)) tails, were first discovered in RNA viruses 40 years ago (Sanger et al., 1976). CircRNAs are functionally important for these viruses to generate multiple genomic copies by rolling circle amplification of the RNA genome with host DNA-dependent RNA polymerases. Later, a small number of additional endogenous circRNAs were revealed in unicellular eukaryotes (Grabowski et al., 1981), archaea (Kjems \& Garrett, 1988) and higher eukaryotes (Capel et al., 1993; Cocquerelle et al., 1993). Whereas, it was not until the raise of Next-generation sequencing (NGS) technology and bioinformatics that numerous circRNAs were discovered in highly diverged eukaryotic organisms (Guo et al., 2014; Memczak et al., 2013; Rahimi et al., 2019; Westholm et al., 2014).

Studies have suggested a cell type/tissue- specific manner of circRNAs expression, and some of them were expressed across different species (Guo et al., 2014; Jeck et al., 2013; Memczak et al., 2013; Salzman et al., 2013). Based on their origin, circRNAs can be grouped into four 
40 categories: exonic circRNAs, only consisted of exons; intronic circRNAs, only consisted of

41 introns; exonic-intronic circRNAs, consisted of exons and introns; and intergenic circRNAs from

42 intergenic regions (Wang et al., 2016). Unlike linear RNAs, circRNAs show long half-lives and

43 play various biological roles, such as function as microRNA (miRNA) sponges (Hansen et al.,

44 2013), regulating parental gene transcription (Li et al., 2015) and cell proliferation (Bachmayr-

45 Heyda et al., 2015), interacting with RNA-binding proteins (RBPs) (Li et al., 2017), as well as

46 translating proteins (Meng et al., 2017). Previous bioinformatics tools have been raised for large-

47 scale circRNAs identification by using back-splicing junction sites (BSJs) to represent different

48 circRNAs (Gao et al., 2015; Guo et al., 2014; Zhang et al., 2014). However, the functional and

49 evolutionary analyses of circRNAs depends on their full-length sequences. Considering the

50 prevalence of alternative circRNAs processing, such as exon skipping and intron retention (Dong

51 et al., 2017; Gao et al., 2016; You et al., 2015; Zhang et al., 2016a), the aforementioned methods

may provide inaccurate information of circRNA isoforms which have same BSJs but differ in their internal compositions. To solve the above problems, lots of effort has been made for

accurate determination of full-length circRNAs. In this review, we summarize the recent findings on biogenesis of circRNA isoforms, progress on detection methods, and highlight the challenges for further research.

\section{Survey methodology}

58 Article searching was performed in Web of Science, PubMed and Elsevier with the words "alternative splicing", "alternative back-splicing" or "circRNAs enrichment" in combination with the terms "circRNAs" or "full-length circRNAs" in the title and abstract. All studies published both in English and in Chinese were searched with no restriction on the publication period. In addition, the reference lists of the retrieved articles were manually searched to identify potentially relevant studies. We focused mainly on the biogenesis of circRNAs, factors resulting in alternative splicing and experimental and bioinformatic methodologies which are expected to accurately discover full-length circRNAs.

\section{Biogenesis of CircRNAs}

Recent studies unveiled that circRNAs are not only derived from numerous of precursor mRNAs (pre-mRNAs) but also long non-coding RNAs (lncRNAs) (Burd, et al., 2010; Holdt et al., 2016; Huang et al., 2018; Zhang et al., 2019). Two mechanisms have been raised to explain the formation of circRNAs (Fig. 1), which are defined as direct back-splicing and lariat intermediate (exon skipping) (Jeck \& Sharpless, 2014). Broadly speaking, both models involve back-splicing and canonical splicing, but they are notably different in which step happens first (Chen \& Yang, 2015).

\section{Direct back-splicing}

Complementary sequences across long flanking introns (Liang \& Wilusz, 2014; Zhang et al., 2014) or the dimerization of RBPs (Ashwal-Fluss et al., 2014; Conn et al., 2015; Li et al., 2017) can facilitate 'direct back-splicing' by bridging the splice donor site into close proximity to the acceptor site (Fig. 1A) (Chen \& Yang, 2015). Therefore, the branch point upstream of a circularized exon is able to attack the downstream splice donor site, resulting in a pre-mRNA 
80

81

82

83

84

85

86

87

88

89

90

91

92

93

94

95

96

97

98

99

100

101

102

103

104

105

106

107

108

109

110

111

112

113

114

115

116

117

118

119

intermediate containing a 2', 5'-phosphodiester linkage. Subsequently, the upstream splice acceptor is attacked by the free $3^{\prime}$ hydroxyl group of the prospectively circRNA to form a circRNA. It is worthwhile noting that one set of double-stranded RBPs, Adenosine deaminase acting on RNA (ADARs), suppress circRNA expression by destabilizing intron pairing interactions (Ivanov et al., 2015).

\section{Lariat intermediate}

Interestingly, inverted repeated elements flanking the circle-forming exons are widespread in mammals but rare in lower eukaryotes. Lariat intermediate mechanism has been proposed in the literature accounting for producing circRNAs (Fig. 1B, left). Using fission yeast (Schizosaccharomyces pombe) as a model system, researchers found that mrps16 gene produced a circRNA by forming an exon-containing lariat firstly (Barrett et al., 2015). In this model, canonical splicing appears first, forming a lariat precursor (Eger et al., 2018). And then internal back-splicing take place, which result in a double lariat molecular and a circRNA.

According to this approach, alternative exons or introns are excised from the pre-mRNA as exon-intron(s)-exon intermediate molecules. Lariat structure is not permanently stable for the reason that the 2' to 5' phosphodiester bond will be recognized and debranched specifically to linear form by debranching endonucleases (Hesselberth, 2013). However, some lariats are relatively stable and may probably escape from debranching to forming intronic circRNAs (ciRNAs), when contain consensus RNA sequences (a 7-nt GU-rich sequence near the 5' splice site and an 11-nt C-rich sequence near the branch point). These ciRNAs are distinguished from exonic circRNAs by a 2' to 5' phosphodiester bond (Fig. 1B, right) (Lasda \& Parker, 2014; Zhang et al., 2013).

\section{Alternative Splicing within CircRNAs}

Alternative splicing (AS) in protein-coding or non-coding genes has a dramatic impact on in cellular differentiation and organismal development. Similar to linear isoforms, circRNAs generated from multi-exon genes are alternatively spliced as well, and may further plays important roles in the transcriptome (Feng et al.,2019; Gao et al., 2016). AS events in circRNAs fall into two categories: circRNAs with alternative BSJs (ABSJs) and circRNA with same BSJs but distinct internal compositions (Fig. 2).

As for ABSJs, a single gene could produce multiple circRNAs which contain different 5' splice donors or 3' splice acceptors (Fig. 2A) (Zhang et al., 2016a). For instance, DNMT3B and XPO1 gene in human produced multiple highly expressed circRNAs through this mechanism.

Experimental methods, such as reverse transcription-PCR (RT-PCR) or RNA-sequencing (RNAseq), are able to validate this phenomenon (Beltrán-García, et al., 2020; Jeck \& Sharpless, 2014). Different from ABSJs, the occurrence of alternative canonical splicing within circRNAs leads to isoforms that have the same BSJs but distinct in internal compositions (Gao et al., 2016, Zhang et al., 2016a). CircRNAs derived in this manner, can be grouped into one of four categories: cassette exon, intron retention, alternative 5' splicing, and alternative 3' splicing (Fig. 2B) (Gao et al., 2016; Jeck et al., 2013; Memczak et al., 2013; Salzman et al., 2013; Zhang et al., 2016a; Zhang et al., 2014). Researchers identified that numerous new exons retained in circRNAs by 
120

121

122

123

124

125

126

127

128

129

130

131

132

133

134

135

136

137

138

139

140

141

142

143

144

145

146

147

148

149

150

151

152

153

154

155

156

157

158

specific alternative canonical splicing. Consistent with this, several previously unannotated exons in the human MED13L locus were discovered by analyzing multiple poly(A)- RNA-seq (Zhang et al., 2016a). Additionally, circRNAs with or without a retained intron generated from CAMSAP1 locus, were confirmed by northern blotting assay (Salzman et al., 2013; Zhang et al., 2014). Similarly, a cassette exon in the human XPO1 locus was proved to be circRNApredominant (Zhang et al., 2014).

Previous researches showed that more than $50 \%$ of the expressed loci could produce two or more circRNA isoforms by AS (Gao et al., 2016; Ji et al., 2019; Ottesen et al., 2019; Rui et al., 2018; Zhang et al., 2016a; Zheng et al., 2019). According to Feng et al., AS events in circRNAs were potentially involved in cancer processes. For instance, in UBAP2L locus, alternative 5' splicing occurred in circRNAs between cancer and adjacent normal tissues (Feng et al., 2019). Similarly, skipping exon event occurred in circRNAs from RAB6A. Therefore, it is important to explore circRNA AS events, and ongoing investigations have begun to focus on factors which were linked to AS, such as circRNA-Rolling Circle Amplification and CIRI-full (Das et al., 2019; Zheng et al., 2019).

\section{The Mechanisms of Alternative Splicing Events}

Since circRNA processing is related to transcription and pre-mRNA splicing, circularization is presumably influenced by both cis-regulatory elements and trans-acting factors, for instance, spliceosome assembly (Ashwal-Fluss et al., 2014; Liang et al., 2017; Starke et al., 2015), topological effects due to intronic sequence (Chen, 2016; Zhang et al., 2014) and combinatorial effects of RBPs (Ashwal-Fluss et al., 2014; Conn et al., 2015). Remarkably, the mechanism of back-splicing has been determined to some extent and several factors related to generation of different circRNA isoforms are listed below.

\section{Competition of reverse complementary sequences}

Considering that most of circRNAs are generated after their parent genes have been transcribed completely, the majority of circRNA isoforms may occur post-transcriptionally (Ashwal-Fluss et al., 2014; Zhang et al., 2016b). Reverse complementary sequences flanking the circularized exons, such as abundant Alu elements (Jeck \& Sharpless, 2014), highly conserved mammalianwide interspersed repeat (MIR) sequence (Yoshimoto et al., 2019) or other non-repetitive complementary sequence (Memczak et al., 2013), are efficient to enhance exon circularization by forming paired duplex structures (Liang and Wilusz, 2014). Shorter sequences as long as 30 to 40 nucleotides are even able to promote circRNA biogenesis (Liang and Wilusz, 2014). Once the intronic complementary sequences across the circularized exons were disrupted, no circRNA could be detected at the examined locus (Zhang et al., 2016b).

Theoretically, a series of inverted repeated RNA pairs form different RNA duplexes resulted in multiple circRNA isoforms (Fig. 3, b-c). Moreover, an individual intron can also form RNA pairing which promotes canonical splicing to linear RNA formation (Fig. 3, a). It means that the competition of RNA pairing leads to different splicing products (Ashwal-Fluss et al., 2014; Wilusz \& Jeremy, 2018). Taken together, the endogenous conditions for circRNAs generation 
159

160

161

162

163

164

165

166

167

168

169

170

171

172

173

174

175

176

177

178

179

180

181

182

183

184

185

186

187

188

189

190

191

192

193

194

195

196

197

are very complex, since the number of repetitive elements, the distance between them, and their degree of complementarity all affect the splicing outcome (Zhang et al., 2014).

\section{RNA Binding Proteins}

Various AS events were observed despite of the presumably identical of paired complementary sequences among all tested human samples (Zhang et al., 2016a), which indicated that the regulation of AS is more complicated than we found. Previous study showed that the RNA pairing was influenced by RBPs as well (Ivanov et al., 2015). These proteins bind to specific intron motifs firstly and then bring the donor site closer to the acceptor site (Ashwal-Fluss et al., 2014; Conn et al., 2015). In contrast, negative RBPs will suppress circRNA formation by destabilizing RNA pairing interactions, like ADAR1 (Ivanov et al., 2015; Rybak-Wolf et al., 2015). Differentially expressed RBPs have been reported to mediate pre-mRNA AS in various cell lines, for example, SR and SR-related proteins typically activate splicing (Nilsen et al., 2010). By contrast, hnRNPs are generally thought to repress splicing. The activity of these proteins can be regulated through signaling networks in specific tissues and cell-types (McManus, et al., 2011). Hence, we hypothesize that AS in circRNAs may be regulated by similar mechanism.

\section{Others}

It is well known that pre-mRNA processing is closely correlated with polymerase II (Pol II) transcription, and the transcription elongation rate has obvious effect on the occurrence of splicing events (Bentley, 2014). Flies with decreased elongation capacity of RNA pol II, produced a significantly lower number of circRNAs (Ashwal-Fluss et al., 2014). By applying 4thiouridine ( $4 \mathrm{~s} \mathrm{~s})$ to metabolic tagging of nascent RNAs, Zhang et al. discovered that circRNAproducing genes had higher average transcription elongation rate (Zhang et al., 2016b). In summary, the positive correlation between nascent circRNA generation and Pol II elongation speed indicates that fast elongation favors RNA folding across flanking introns to form circRNAs rather than within introns (Bentley, 2014; Zhang et al., 2016b).

Nevertheless, those factors mentioned above still cannot adequately explain the widespread nature of AS in circRNAs. Additional elements that are responsible for alternative circularization await identification. It is helpful to discover the rules of AS in circRNAs by large-scale identification of full-length circRNAs. Currently, lots of endeavors are focused on the internal structure of circRNAs which further promote their functional characterization and evolutionary analyses.

\section{Current Approaches for Identification of Alternative Splicing}

CircRNAs containing unique BSJs, are differ obviously from their host linear RNA counterparts, and hence the BSJs is critical for their identification. So far, a number of biomedical methods have been raised to identify and quantify circRNAs, including RT-PCR/qPCR, Northern blot analyses (Memczak et al., 2013; Zhang et al., 2016c; Zhang et al., 2013), and circRNA microarray (Li et al., 2019; Zhang et al., 2018). Until the development of NGS and bioinformatics tools, the abundant circRNAs were revealed in multiple cell types or tissues. 
198

199

200

201

202

203

204

205

206

207

208

209

210

211

212

213

214

215

216

217

218

219

220

221

222

223

224

225

226

227

228

229

230

231

232

233

234

235

236

237

Many bioinformatic analyzing tools combine all known mRNA exons in a sequential order to represent putative full-length circRNA, which bases on an unsupported assumption that circRNAs possess identical composition with their linear counterparts (Guo et al., 2014; Zhang et al., 2014). For example, circular(CIRC)explorer (Zhang et al., 2014), MapSplice (Wang et al., 2010), circRNA_Finder (Xing \& Liu, 2014), Circular RNA identification (CIRI) (Gao et al., 2015), Find_circ (Memczak et al., 2013) and others, are developed for circRNA identification but not assembly of full-length circRNAs.

Given the fact that distinct splicing leads to differential components of exons and/or introns, using BSJs only to represent subsets of circRNA variants greatly limits our understanding of biogenesis, functions and evolution of circRNAs among species. Due to the technically challenging, the aforementioned tools are unable to explore the internal sequence of circRNAs accurately or distinguish AS in linear RNAs from circRNAs derived from the same gene. Recently, a number of efforts have been made to address this challenge.

\section{CircRNA enrichment methods}

Current studies show that circRNAs abundance is approximately $\leq 10 \%$ of their corresponding linear RNA (Salzman et al., 2013), and the estimation of circRNA isoforms with lower expression level probably be biased. Therefore, circRNA enrichment is needed prior to RNA library construction and sequencing. Due to the great variability length of circRNAs, it is hard to separate them from other RNA species by size or electrophoretic mobility. Owing to the covalently closed structure, most circRNAs show higher tolerance to Ribonuclease R (RNase R) digestion in comparison with linear RNAs. Nevertheless, utilizing RNase $\mathrm{R}$ alone is not sufficient to remove RNAs lacking 3' overhangs. Therefore, researchers pretreated the RNA samples by combining ribosome RNA (rRNA) depletion with RNase R treatment (Jeck \& Sharpless, 2014) or depletion of poly(A)+ RNA to ensure that sequencing reads are generated from bona fide circRNAs.

Whereas, some linear RNAs without sufficiently long single-stranded 3' overhangs, such as small noncoding RNAs, are naturally resistant to RNase R (Vincent \& Deutscher, 2006). Besides, many polyadenylated mRNAs with complex structures, especially G-quadruplex (G4) structures, are also poor substrates for RNase R, as the enzyme stalls in the body of these transcripts (Panda et al., 2017; Xiao \& Wilusz, 2019). Considering that the expression of circRNAs is often lower than the linear counterparts, even a small amount of undigested linear RNAs may surpass the abundant of cognate circRNAs (Panda et al., 2017). To resolve this major obstacle, Panda et al. raised another approach to isolate high-purity circRNA termed RNase $\mathrm{R}$ treatment followed by Polyadenylation and poly(A)+ RNA Depletion (RPAD) (Fig. 4A) (Panda et al., 2017; Pandey et al., 2019). After RNase R treatment, small RNAs were decreased to 10$50 \%$ of the remaining by polyadenylation and poly(A)+ RNA depletion. In an alternative manner, Xiao et al. found that RNase R could digest linear RNAs more efficiently by using $\mathrm{Li}^{+}$ instead of K+ (which stabilizes G4s) in the reaction buffer (Xiao \& Wilusz, 2019). Drawing inspiration from the RPAD method, purer circRNAs can be isolated by coupling A-tailing with RNase R in LiCl-containing buffer (Fig. 4B) (Xiao \& Wilusz, 2019). 
238 Compared with conventional models of circRNA enrichment using rRNA depletion kits of 239 model organisms, the other two methods can be used in non-model organisms for efficient 240 removal of linear RNAs regardless of the RNA sequences. A noteworthy drawback of RPAD is 241 that quantification of circRNA abundance may not be strictly accurate due to involvement of 242 many enzymatic and RNA isolation steps. Furthermore, according to specific experiment 243 purpose, RPAD protocol was modified via adding rRNA depletion step before RNase R 244 treatment (Rahimi et al., 2019). It is worth noticing that extensive purification steps will cause 245 significant biases on circRNA abundance.

\section{Genome-wide annotation of full-length circRNAs}

247 At present, a combination of RNA-seq analysis with bioinformatic survey has been widely used

248

249

250

251

252

253

254

255

256

257

258

259

260

261

262

263

264

265

266

267

268

269

270

271

272

273

274

275

276

277

for large-scale determination of circRNAs. Moreover, various algorithms have been raised to analyse sequencing information generated from different platforms.

\section{Next-generation sequencing methods}

Studies have characterized circular variants arising from one host-gene with ABSJs sites (Jeck et al., 2013; Zhang et al., 2014), and these isoforms can be identified by existing circRNA detection algorithms (Peng et al., 2020). In order to identify circRNA isoforms with the same BSJs, more efficient and stringent tool have been developed and listed in Table 1.

CIRI-AS has been designed to investigate internal components of circRNAs for the first time (Gao et al., 2016). This algorithm is based on reconstructing circRNA exons (cirexons) routes and clustering alternatively spliced cirexons, and could be applied to majority of current available RNA-seq data. Likewise, FUCHS focuses on the AS events within same circle boundaries by analyzing long sequencing reads (typically $>150$ bp) (Metge et al., 2017). In an alternative manner, CIRCexplorer2 predicts AS in circRNAs by comparing data sets between poly(A)+ and poly(A)- RNA-seq (Zhang et al., 2016a). Compared with CIRI-AS, CircSplice could detect more AS events and provide the comparison function of different samples (Feng et al., 2019). According to Ye et al, a bioinformatics pipeline named circseq cup was developed to assemble full-length circRNAs, which utilized fusion junction sites and their corresponding paired-end RNA-seq reads (Ye et al., 2017). The length of circRNAs identified by this approach relied on the read length and sequencing library length. As for CircAST, it is developed for reconstruction and quantification of circRNA variants. Additionally, it shows better performance on variable read lengths (from $75 \mathrm{bp}$ to $125 \mathrm{bp}$ ). However, as an annotated-based method, CircAST may miss intronic or intergenic circRNAs (Wu et al., 2019). CIRI-full reconstructs fulllength circRNAs by combining BSJ and reverse overlap (RO) features and facilitates the identification of low-abundance circRNAs. Meanwhile, it is more suitable for longer sequencing reads ( $>250$ or $300 \mathrm{bp}$ ) (Zheng et al., 2019). Compared with previous methods, the main advantage is that CIRI-full uses unfragmented RNA samples for library preparation.

In summary, these tools have different advantages in detection of circRNA isoforms. Most notably, CIRI-full provides precise sequence of circRNAs ( $\leq 600 \mathrm{bp}$ ) by using cDNA libraries without fragmentation step. On the contrary, the majority of algorithms provides indirect data of circRNAs but perform well even using short read sequencing data. However, they all face an

Peer) reviewing PDF | (2020:07:50735:1:1:NEW 17 Aug 2020) 
278 inherent challenge that the reconstruction of circRNAs relies on the read length as well as the 279 insertion sizes of cDNA libraries (Ye et al., 2017), so they are limited to small circRNAs and 280 biased on prediction of large circRNAs (Gao et al., 2016).

281 Long-read sequencing methods

282 According to the aforementioned that read length is the key determinant of full-length circRNAs 283 validation, the utilization of third-generation sequencing (TGS) technologies, such as PacBio 284 long read sequencing, is likely to identify circRNA variants accurately. By using Oxford 285 Nanopore Technology (ONT), Rahimi et al. performed global examination of full-length 286 circRNAs in human and mouse brains (Rahimi et al., 2019). According to this study, the 287 enriched circRNA pool was linearized and re-polyadenylated prior to library preparation. This 288 study, to some degree, circumvents the limitation of NGS methods and provides a fast and 289 reliable method of circRNA reconstruction. However, its greatest drawback is that fragmentation 290 of circRNAs will probably influence the accuracy of subsequent assembly even though the 291 linearization process has been optimized.

292

293

294

295

296

297

298

299

300

301

302

303

304

305

306

307

308

309

310

311

312

313

314

315

316

317

\section{Experimental validation of full-length circRNAs by Rolling Circle Amplification}

Compared with bioinformatics reconstruction, experimental approaches provide precise sequence of specific circRNAs most intuitively. Hence, follow-up experimental validation for assembled circRNAs may then be applied to further strengthen the case for existence of many circRNAs. As reverse transcriptase has potential strand displacement activity (Kelleher, 1998), it allows the displacement of any complementary sequence hybridized downstream to produce many copies of the same template (Acevedo et al., 2014).

Owing to the unique structure of circRNAs, multiple rounds of reverse transcription could produce double or triple sized products (Barrett et al., 2015; Danan et al., 2012; Starostina et al., 2004). You et al., for the first time, detected the full-length of circRNAs by sequencing of rolling circle cDNA products on PacBio platform (You et al., 2015). Through creating circular-derived PCR products with divergent primers, Hirsch et al. combined ONT sequencing with a PCRbased approach to gain insight into the internal structure of circNPM1 (Hirsch et al., 2017). Moreover, Das et al. presented a novel method, named circRNA-Rolling Circle Amplification (circRNA-RCA), aiming to distinguish circRNA variants containing the same BSJ site but distinct in internal sequence (Fig. 5). In this method, a forward primer spanning the BSJ sequence and a reverse primer exactly upstream of the forward primer were used to produce tandem-repeat cDNA amplicons with the supplementation of RNase H-minus reverse transcriptase (Das et al., 2019). However, the main drawback of these methods is that only a handful of circRNAs can be examined and the sensitivity and /or specificity is not satisfactory.

\section{Conclusions}

Recent advances have revealed partial factors related to the AS events within circRNAs. However, the regulation of AS in endogenous conditions is far more complex than we have discovered and requires further investigation. To some extent, identification of their full-length sequence contributes to further understanding the regulation and function of AS. In light of the issues, novel bioinformatics tools have been raised to reconstruct circRNA isoforms such as

Peer) reviewing PDF | (2020:07:50735:1:1:NEW 17 Aug 2020) 
318 CIRI-AS and CircAST. However, they provide indirect data of circRNAs due to the

319 fragmentation step before library preparation. Despite the utilization of non-fragmented samples,

320 CIRI-full is difficult to completely recover large circRNAs which is limited by the short-read

321 length of NGS. Remarkably, experimental approaches like circRNA-RCA provide precise

322 sequence of specific circRNAs, while only a limited subset of circRNAs could be validated.

323 In summary, there is no competent method that is applicable for large-scale circRNAs detection

324 with high accuracy. We believe that the combination of rolling circle amplification with TGS

325 technologies is a promising method for full-length circRNAs identification. And with the

326 development of methodologies, new insights will be provided on understanding roles of

327 circRNAs and previous unknown biological phenomena in the coming years.

328

329 Declaration of competing interest

330 The authors declare no conflict of interest.

331

332

333

334

335

336

337

338

339

340

341

342

343

344

345

346

347

348

349

350

351

352

353

\section{Author Contributions}

Xiaohan Li: writing — original draft preparation. Bing Zhang, Fuyu Li, Kequan Yu and Yunfei Bai: writing—review and editing.

\section{References}

Acevedo A, Brodsky L, and Andino R. 2014. Mutational and fitness landscapes of an RNA virus revealed through population sequencing. Nature 505:686-690. DOI: 10.1038/nature12861.

Ashwal-Fluss R, Meyer M, Pamudurti NR, Ivanov A, Bartok O, Hanan M, Evantal N, Memczak S, Rajewsky N, and Kadener S. 2014. circRNA biogenesis competes with pre-mRNA splicing. Molecular cell 56:55-66. DOI: 10.1016/j.molcel.2014.08.019.

Bachmayr-Heyda A, Reiner AT, Auer K, Sukhbaatar N, Aust S, Bachleitner-Hofmann T, Mesteri I, Grunt TW, Zeillinger R, and Pils D. 2015. Correlation of circular RNA abundance with proliferation-exemplified with colorectal and ovarian cancer, idiopathic lung fibrosis, and normal human tissues. Scientific reports 5:8057. DOI: 10.1038/srep08057.

Barrett SP, Wang PL, and Salzman J. 2015. Circular RNA biogenesis can proceed through an exon-containing lariat precursor. elife 4:e07540. DOI: 10.7554/eLife.07540.001.

Beltrán-García J, Osca-Verdegal R, Nacher-Sendra E, Pallardó FV, García-Giménez JL. 2020. Circular RNAs in Sepsis: Biogenesis, Function, and Clinical Significance. Cell 9:1544. DOI: 10.3390/cells9061544.

Bentley DL. 2014. Coupling mRNA processing with transcription in time and space. Nature Reviews Genetics 15:163-175. DOI: 10.1038/nrg3662. 
354

355

356

357

358

359

360

361

362

363

364

365

366

367

368

369

370

371

372

373

374

375

376

377

378

379

380

381

382

383

384

385

386

387

388

389

390

391

392

393

Burd CE, Jeck WR, Liu Y, Sanoff HK, Wang Z, Sharpless NE. 2010. Expression of Linear and Novel Circular Forms of an INK4/ARF-Associated Non-Coding RNA Correlates with Atherosclerosis Risk. Plos Genetics 6:e1001233. DOI: 10.1371/journal.pgen.1001233. Capel B, Swain A, Nicolis S, Hacker A, Walter M, Koopman P, Goodfellow P, and LovellBadge R. 1993. Circular transcripts of the testis-determining gene Sry in adult mouse testis. Cell 73:1019-1030. DOI: 10.1016/0092-8674(93)90279-Y.

Chen L-L. 2016. The biogenesis and emerging roles of circular RNAs. Nature Reviews Molecular Cell Biology 17:205-211. DOI: 10.1038/nrm.2015.32.

Chen L-L, and Yang L. 2015. Regulation of circRNA biogenesis. RNA biology 12:381-388. DOI: 10.1080/15476286.2015.1020271.

Cocquerelle C, Mascrez B, Hetuin D, and Bailleul B. 1993. Mis-splicing yields circular RNA molecules. The FASEB Journal 7:155-160. DOI: 10.1096/fasebj.7.1.7678559.

Conn SJ, Pillman KA, Toubia J, Conn VM, Salmanidis M, Phillips CA, Roslan S, Schreiber AW, Gregory PA, and Goodall GJ. 2015. The RNA binding protein quaking regulates formation of circRNAs. Cell 160:1125-1134. DOI: 10.1016/j.cell.2015.02.014.

Danan M, Schwartz S, Edelheit S, and Sorek R. 2012. Transcriptome-wide discovery of circular RNAs in Archaea. Nucleic acids research 40:3131-3142. DOI: 10.1093/nar/gkr1009.

Das A, Rout PK, Gorospe M, and Panda AC. 2019. Rolling Circle cDNA Synthesis Uncovers Circular RNA Splice Variants. International journal of molecular sciences 20:3988. DOI: 10.3390/ijms20163988.

Dong R, Ma X-K, Chen L-L, and Yang L. 2017. Increased complexity of circRNA expression during species evolution. RNA biology 14:1064-1074. DOI: 10.1080/15476286.2016.1269999.

Eger N, Schoppe L, Schuster S, Laufs U, and Boeckel J-N. 2018. Circular RNA Splicing. In: Xiao J, ed. Circular RNAs: Biogenesis and Functions. Singapore: Springer, 41-52.DOI: 10.1007/978-981-13-1426-1_4.

Feng J, Chen K, Dong X, Xu XL, Jin YX, Zhang XY, Chen WB, Han YJ, Shao L, Gao Y, and He CJ. 2019. Genome-wide identification of cancer-specific alternative splicing in circRNA. Molecular Cancer 18. DOI: 10.1186/s12943-019-0996-0.

Gao Y, Wang J, and Zhao F. CIRI: an efficient and unbiased algorithm for de novo circular RNA identification. Genome biology 16:4. DOI: 10.1186/s13059-014-0571-3.

Gao Y, Wang J, Zheng Y, Zhang J, Chen S, and Zhao F. 2016. Comprehensive identification of internal structure and alternative splicing events in circular RNAs. Nature communications 7:12060. DOI: $10.1038 /$ ncomms 12060 .

Grabowski PJ, Zaug AJ, and Cech TR. 1981. The intervening sequence of the ribosomal RNA precursor is converted to a circular RNA in isolated nuclei of Tetrahymena. Cell 23:467476. DOI: 10.1016/0092-8674(81)90142-2.

Guo JU, Agarwal V, Guo H, and Bartel DP. 2014. Expanded identification and characterization of mammalian circular RNAs. Genome biology 15:409. DOI: 10.1186/PREACCEPT1176565312639289 . 
394

395

396

397

398

399

400

401

402

403

404

405

406

407

408

409

410

411

412

413

414

415

416

417

418

419

420

421

422

423

424

425

426

427

428

429

430

431

432

Hansen TB, Jensen TI, Clausen BH, Bramsen JB, Finsen B, Damgaard CK, and Kjems J. 2013. Natural RNA circles function as efficient microRNA sponges. Nature 495:384-388. DOI: 10.1038/nature11993.

Hesselberth JR. 2013. Lives that introns lead after splicing. Wiley Interdisciplinary Reviews: RNA 4:677-691. DOI: 10.1002/wrna.1187.

Hirsch S, Blätte TJ, Grasedieck S, Cocciardi S, Rouhi A, Jongen-Lavrencic M, Paschka P, Krönke J, Gaidzik VI, and Döhner H. 2017. Circular RNAs of the nucleophosmin (NPM1) gene in acute myeloid leukemia. Haematologica 102:2039-2047. DOI: 10.3324/haematol.2017.172866.

Holdt LM, Stahringer A, Sass K, Pichler G, Kulak NA, Wilfert W, Kohlmaier A, Herbst A, Northoff BH, and Nicolaou A. 2016. Circular non-coding RNA ANRIL modulates ribosomal RNA maturation and atherosclerosis in humans. Nature communications 7:12429. DOI: 10.1038/ncomms 12429.

Huang M-S, Zhu T, Li L, Xie P, Li X, Zhou H-H, and Liu Z-Q. 2018. LncRNAs and CircRNAs from the same gene: masterpieces of RNA splicing. Cancer letters 415:49-57. DOI: 10.1016/j.canlet.2017.11.034.

Ivanov A, Memczak S, Wyler E, Torti F, Porath HT, Orejuela MR, Piechotta M, Levanon EY, Landthaler M, and Dieterich C. 2015. Analysis of intron sequences reveals hallmarks of circular RNA biogenesis in animals. Cell reports 10:170-177. DOI: 10.1016/j.celrep.2014.12.019.

Jeck WR, and Sharpless NE. 2014. Detecting and characterizing circular RNAs. Nature biotechnology 32:453-461. DOI: 10.1038/nbt.2890.

Jeck WR, Sorrentino JA, Wang K, Slevin MK, Burd CE, Liu J, Marzluff WF, and Sharpless NE. 2013. Circular RNAs are abundant, conserved, and associated with ALU repeats. Rna 19:141-157. DOI: 10.1261/rna.035667.112.

Ji PF, Wu WY, Chen S, Zheng Y, Zhou L, Zhang JY, Cheng H, Yan J, Zhang SG, Yang PH, and Zhao FQ. 2019. Expanded Expression Landscape and Prioritization of Circular RNAs in Mammals. Cell reports 26:3444-3480. DOI: 10.1016/j.celrep.2019.02.078.

Kelleher, and D. C. Characterization of RNA Strand Displacement Synthesis by Moloney Murine Leukemia Virus Reverse Transcriptase. Journal of Biological Chemistry 273:99769986. DOI: $10.1074 /$ jbc.273.16.9976.

Kjems J, and Garrett RA. 1988. Novel splicing mechanism for the ribosomal RNA intron in the archaebacterium Desulfurococcus mobilis. Cell 54:693-703. DOI: 10.1016/S00928674(88)80014-X.

Lasda E, and Parker R. 2014. Circular RNAs: diversity of form and function. Rna 20:1829-1842. DOI: $10.1261 /$ rna.047126.114.

Li S, Teng S, Xu J, Su G, Zhang Y, Zhao J, Zhang S, Wang H, Qin W, and Lu ZJ. 2019. Microarray is an efficient tool for circRNA profiling. Briefings in bioinformatics 20:14201433. DOI: $10.1093 / \mathrm{bib} / \mathrm{bby} 006$. 
433

434

435

436

437

438

439

440

441

442

443

444

445

446

447

448

449

450

451

452

453

454

455

456

457

458

459

460

461

462

463

464

465

466

467

468

469

470

471

Li X, Liu C-X, Xue W, Zhang Y, Jiang S, Yin Q-F, Wei J, Yao R-W, Yang L, and Chen L-L. 2017. Coordinated circRNA biogenesis and function with NF90/NF110 in viral infection. Molecular cell 67:214-227. DOI: 10.1016/j.molcel.2017.05.023.

Li Z, Huang C, Bao C, Chen L, Lin M, Wang X, Zhong G, Yu B, Hu W, and Dai L. 2015. Exonintron circular RNAs regulate transcription in the nucleus. Nature structural \& molecular biology 22:256-264. DOI: 10.1038/nsmb0217-194a.

Liang D, Tatomer DC, Luo Z, Wu H, Yang L, Chen L-L, Cherry S, and Wilusz JE. 2017. The output of protein-coding genes shifts to circular RNAs when the pre-mRNA processing machinery is limiting. Molecular cell 68:940-954. DOI: 10.1016/j.molcel.2017.10.034.

Liang D, and Wilusz JE. 2014. Short intronic repeat sequences facilitate circular RNA production. Genes \& development 28:2233-2247. DOI: 10.1101/gad.251926.114.

McManus CJ, Graveley BR. 2011. RNA structure and the mechanisms of alternative splicing. Current Opinion in Genetics \& Development. 21:373-379. DOI: 10.1016/j.gde.2011.04.001.

Memczak S, Jens M, Elefsinioti A, Torti F, Krueger J, Rybak A, Maier L, Mackowiak SD, Gregersen LH, and Munschauer M. 2013. Circular RNAs are a large class of animal RNAs with regulatory potency. Nature 495:333-338. DOI: 10.1038/nature11928.

Meng X, Chen Q, Zhang P, and Chen M. 2017. CircPro: an integrated tool for the identification of circRNAs with protein-coding potential. Bioinformatics 33:3314-3316. DOI: 10.1093/bioinformatics/btx446.

Metge F, Czaja-Hasse LF, Reinhardt R, and Dieterich C. 2017. FUCHS - towards full circular RNA characterization using RNAseq. PeerJ 5:e2934. DOI: 10.7717/peerj.2934.

Nilsen, Timothy, W., Graveley, Brenton, and R. 2010. Expansion of the eukaryotic proteome by alternative splicing. Nature 463:457-463. DOI: 10.1038/nature08909.

Ottesen EW, Luo D, Seo J, Singh NN, and Singh RN. 2019. Human Survival Motor Neuron genes generate a vast repertoire of circular RNAs. Nucleic acids research 47:2884-2905. DOI: $10.1093 / \mathrm{nar} / \mathrm{gkz} 034$.

Panda AC, De S, Grammatikakis I, Munk R, Yang X, Piao Y, Dudekula DB, Abdelmohsen K, and Gorospe M. 2017. High-purity circular RNA isolation method (RPAD) reveals vast collection of intronic circRNAs. Nucleic acids research 45:e116-e116. DOI: 10.1093/nar/gkx297.

Pandey PR, Rout PK, Das A, Gorospe M, and Panda AC. 2019. RPAD (RNase R treatment, polyadenylation, and poly(A) plus RNA depletion) method to isolate highly pure circular RNA. Methods 155:41-48. DOI: 10.1016/j.ymeth.2018.10.022.

Peng Z, Xiao-Ou Z, Tingting J, Lingling C, Xiao H, Qi L, Dan L, Aiping L, Yan L, and Wen X. 2020. Comprehensive identification of alternative back-splicing in human tissue transcriptomes. Nucleic acids research 48:4. DOI: 10.1093/nar/gkaa005.

Rahimi K, Veno MT, Dupont DM, and Kjems J. 2019. Nanopore sequencing of full-length circRNAs in human and mouse brains reveals circRNA-specific exon usage and intron retention. BioRxiv:567164. DOI: 10.1101/567164. 
472 Rui, Dong, Xu-Kai, Ma, Guo-Wei, Li, and Yang. 2018. CIRCpedia v2: An Updated Database for

473

474

475

476

477

478

479

480

481

482

483

484

485

486

487

488

489

490

491

492

493

494

495

496

497

498

499

500

501

502

503

504

505

506

507

508

509

510

511 Comprehensive Circular RNA Annotation and Expression Comparison. Genomics Proteomics \& Bioinformatics 16:226-233. DOI: 10.1016/j.gpb.2018.08.001.

Rybak-Wolf A, Stottmeister C, Glažar P, Jens M, Pino N, Giusti S, Hanan M, Behm M, Bartok O, and Ashwal-Fluss R. 2015. Circular RNAs in the mammalian brain are highly abundant, conserved, and dynamically expressed. Molecular cell 58:870-885. DOI: 10.1016/j.molcel.2015.03.027.

Salzman J, Chen RE, Olsen MN, Wang PL, and Brown PO. 2013. Cell-type specific features of circular RNA expression. PLoS genetics 9:e1003777. DOI: 10.1371/journal.pgen.1003777.

Sanger HL, Klotz G, Riesner D, Gross HJ, and Kleinschmidt AK. 1976. Viroids are singlestranded covalently closed circular RNA molecules existing as highly base-paired rod-like structures. Proceedings of the National Academy of Sciences 73:3852-3856. DOI: 10.1073/pnas.73.11.3852.

Starke S, Jost I, Rossbach O, Schneider T, Schreiner S, Hung L-H, and Bindereif A. 2015. Exon circularization requires canonical splice signals. Cell reports 10:103-111. DOI: 10.1016/j.celrep.2014.12.002.

Starostina NG, Marshburn S, Johnson LS, Eddy SR, Terns RM, and Terns MP. 2004. Circular box C/D RNAs in Pyrococcus furiosus. Proceedings of the National Academy of Sciences 101:14097-14101. DOI: 10.1073/pnas.0403520101.

Vincent HA, and Deutscher MP. 2006. Substrate recognition and catalysis by the exoribonuclease RNase R. Journal of Biological Chemistry 281:29769-29775. DOI: 10.1074/jbc.m606744200.

Wang F, Nazarali AJ, and Ji S. 2016. Circular RNAs as potential biomarkers for cancer diagnosis and therapy. American journal of cancer research 6:1167. PMID: 27429839.

Wang K, Singh D, Zeng Z, Coleman SJ, Huang Y, Savich GL, He X, Mieczkowski P, Grimm SA, and Perou CM. 2010. MapSplice: accurate mapping of RNA-seq reads for splice junction discovery. Nucleic acids research 38:e178-e178. DOI: 10.1093/nar/gkq622.

Westholm JO, Miura P, Olson S, Shenker S, Joseph B, Sanfilippo P, Celniker SE, Graveley BR, and Lai EC. 2014. Genome-wide analysis of drosophila circular RNAs reveals their structural and sequence properties and age-dependent neural accumulation. Cell reports 9:1966-1980. DOI: 10.1016/j.celrep.2014.10.062.

Wilusz, and Jeremy E. 2018. A $360^{\circ}$ view of circular RNAs: From biogenesis to functions. Wiley Interdisciplinary Reviews-rna 9:e1478. DOI: 10.1002/wrna.1478.

Wu J, Li Y, Wang C, Cui Y, Xu T, Wang C, Wang X, Sha J, Jiang B, Wang K, Hu Z, Guo X, and Song X. 2019. CircAST: Full-length Assembly and Quantification of Alternatively Spliced Isoforms in Circular RNAs. Genomics Proteomics \& Bioinformatics 17:522-534. DOI: $10.1016 /$ j.gpb.2019.03.004.

Xiao M-S, and Wilusz JE. 2019. An improved method for circular RNA purification using RNase R that efficiently removes linear RNAs containing G-quadruplexes or structured 3' ends. Nucleic acids research 47:8755-8769. DOI: 10.1093/nar/gkz576.

Peer] reviewing PDF | (2020:07:50735:1:1:NEW 17 Aug 2020) 
512 Xing F, and Liu R. 2014. Circrnafinder: A tool for identifying circular RNAs using RNA-Seq 513 data. The 6th International Conference on Bioinformatics and Computational Biology 514 (BICOB-2014).

515 Ye C-Y, Zhang X, Chu Q, Liu C, Yu Y, Jiang W, Zhu Q-H, Fan L, and Guo L. 2017. Full-length 516 sequence assembly reveals circular RNAs with diverse non-GT/AG splicing signals in rice. $517 \quad$ RNA biology 14:1055-1063. DOI: 10.1080/15476286.2016.1245268.

518 Yoshimoto R, Rahimi K, Hansen T, Kjems J, and Mayeda A. 2019. Biosynthesis of Circular 519 RNA ciRS-7/CDR1as Is Mediated by Mammalian-Wide Interspersed Repeats (MIRs). 520 BioRxiv:411231. DOI: 10.1101/411231.

521 You X, Vlatkovic I, Babic A, Will T, Epstein I, Tushev G, Akbalik G, Wang M, Glock C, and 522 Quedenau C. 2015. Neural circular RNAs are derived from synaptic genes and regulated by

523

524

525

526

527

528

529

530

531

532

533

534

535

536

537

538

539

540

541

542

543

544

545

546

547 development and plasticity. Nature neuroscience 18:603-610. DOI: 10.1038/nn.3975.

Zhang L, Hu J, Li J, Yang Q, Hao M, and Bu L. 2019. Long noncoding RNA LINC-PINT inhibits non-small cell lung cancer progression through sponging miR-218-5p/PDCD4. Artificial cells, nanomedicine, and biotechnology 47:1595-1602. DOI: 10.1080/21691401.2019.1605371.

Zhang S, Zeng X, Ding T, Guo L, Li Y, Ou S, and Yuan H. 2018. Microarray profile of circular RNAs identifies hsa_circ_0014130 as a new circular RNA biomarker in non-small cell lung cancer. Scientific reports 8:1-11. DOI: 10.1038/s41598-018-21300-5.

Zhang X-O, Dong R, Zhang Y, Zhang J-L, Luo Z, Zhang J, Chen L-L, and Yang L. 2016a. Diverse alternative back-splicing and alternative splicing landscape of circular RNAs. Genome research 26:1277-1287. DOI: 10.1101/gr.202895.115.

Zhang X-O, Wang H-B, Zhang Y, Lu X, Chen L-L, and Yang L. 2014. Complementary sequence-mediated exon circularization. Cell 159:134-147. DOI: 10.1016/j.cell.2014.09.001.

Zhang Y, Xue W, Li X, Zhang J, Chen S, Zhang J-L, Yang L, and Chen L-L. 2016b. The biogenesis of nascent circular RNAs. Cell reports 15:611-624. DOI: 10.1016/j.celrep.2016.03.058.

Zhang Y, Yang L, and Chen L-L. 2016c. Characterization of circular RNAs. Long Non-Coding RNAs: Springer, 215-227.DOI: 10.1007/978-1-4939-3378-5_17.

Zhang Y, Zhang X-O, Chen T, Xiang J-F, Yin Q-F, Xing Y-H, Zhu S, Yang L, and Chen L-L. 2013. Circular intronic long noncoding RNAs. Molecular cell 51:792-806. DOI: 10.1016/j.molcel.2013.08.017.

Zheng Y, Ji P, Chen S, Hou L, and Zhao F. 2019. Reconstruction of full-length circular RNAs enables isoform-level quantification. Genome medicine 11:2. DOI: 10.1186/s13073-0190614-1. 
Figure 1

\section{Schematic of circRNA formation.}

(A) The "direct back-splicing" model for the generation of circRNAs. The loop structure can be formed by either complementary sequences across long flanking introns (left) or RBPs (right). The intron sequences are removed or retained to generate Exonic circRNAs or Exonic-intronic circRNAs, respectively. (B) The "lariat intermediate" model for the generation of circRNAs. In this model, canonical splicing appears first, forming a lariat precursor. And then internal back-splicing take place, which result in a double lariat molecular and a circRNA (left). The generation of Intronic circRNAs relies on GU-rich sequences and C-rich sequences to form a lariat intron, followed by tail triming (right).

BP: branchpoint; RBPs: RNA binding proteins.

\section{A Direct back-splicing}

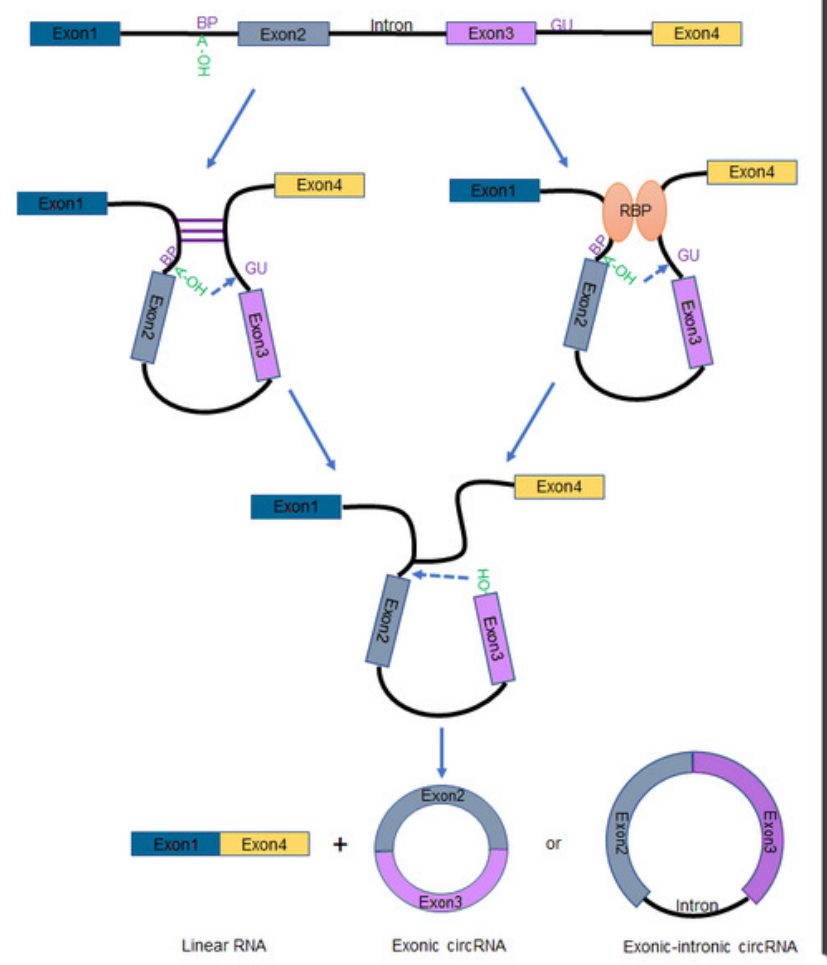

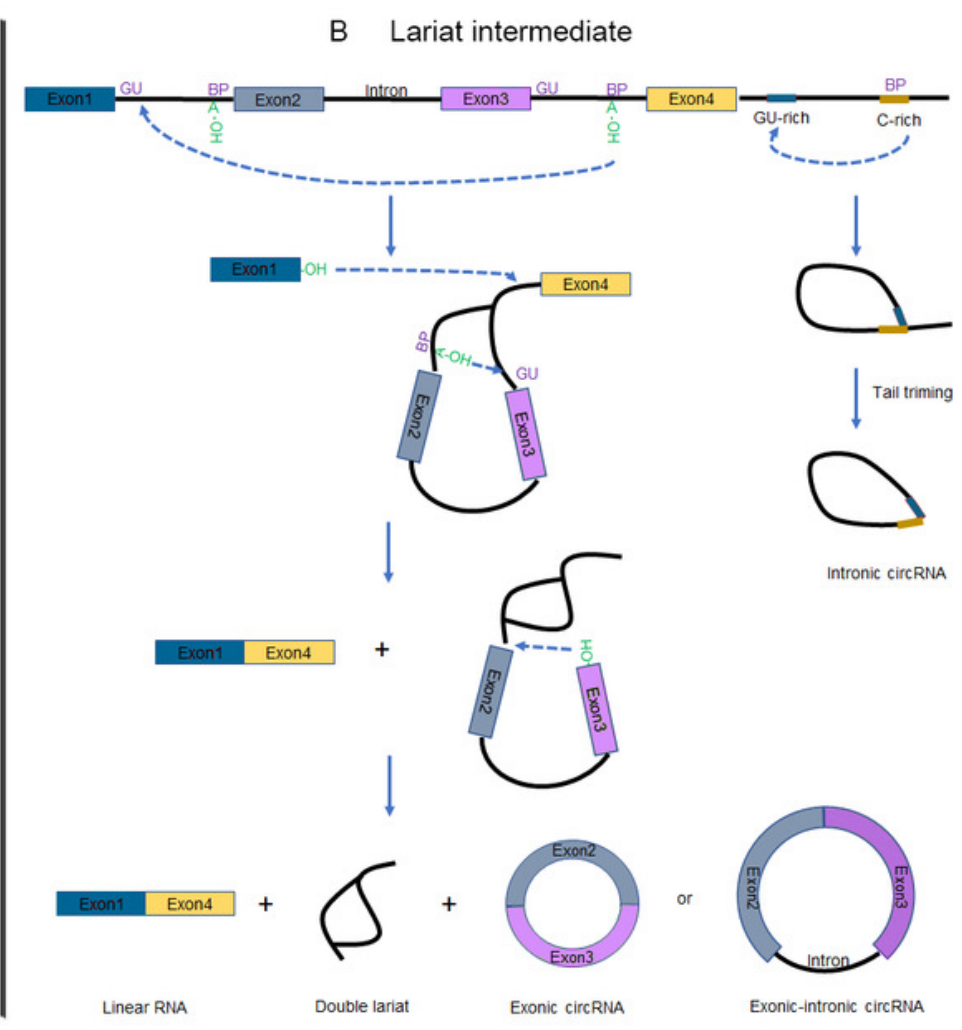


Figure 2

Schematic diagrams of alternative (back-) splicing (Zhang et al., 2016a).

(A) Two types of alternative back-splicing. (B) Four basic types of alternative canonical splicing.

Colored bars: exons; black lines: introns; red polylines: (canonical) collinear splicing; red arc lines: backsplicing (circularization).

Compared with the original image, the font color was changed to black.

A Alternative back-splice site selection

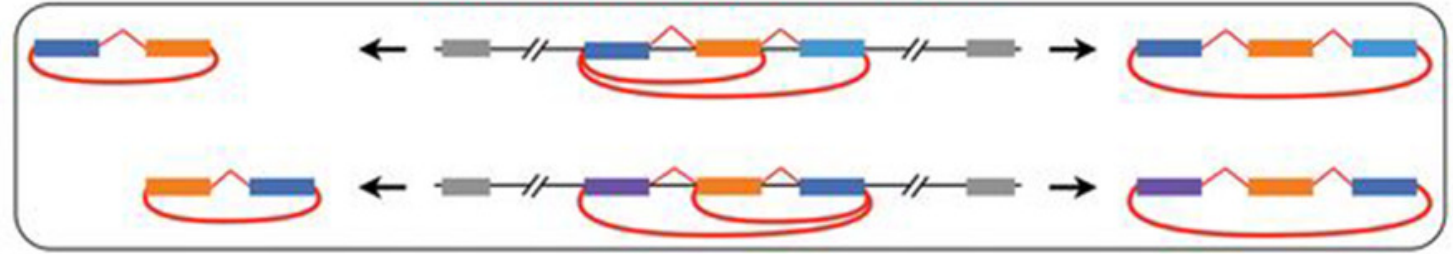

Alternative 5' back-splice site

Alternative 3' back-splice site

B Alternative canonical splice site selection

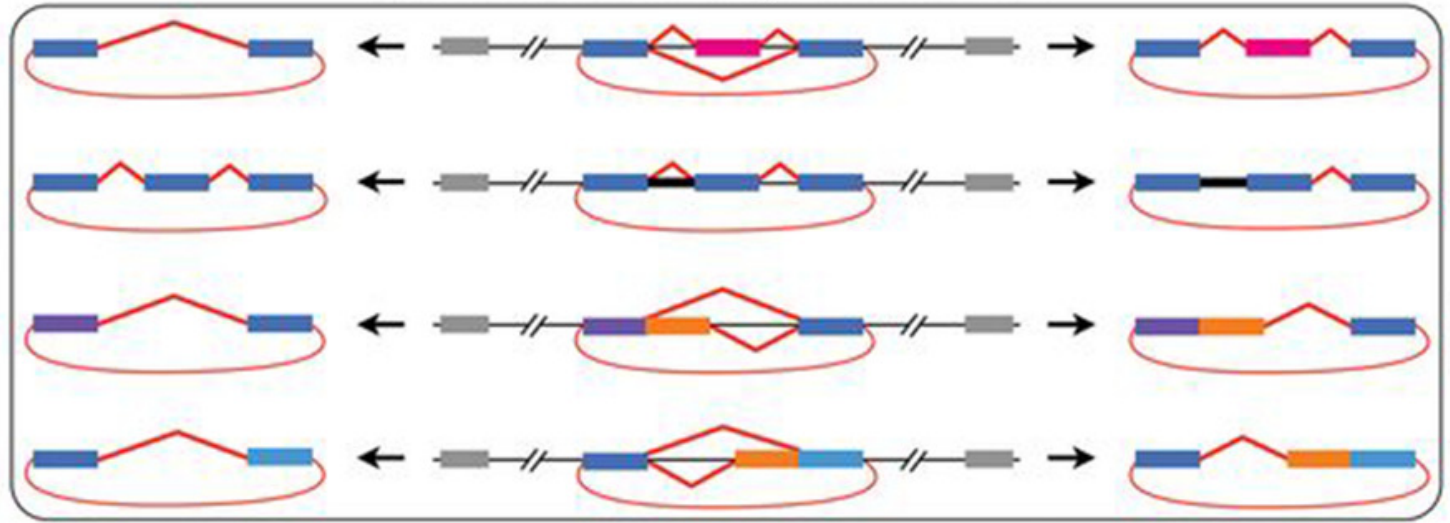

Cassette exon

Intron retention

Alternative 5' splice site

Alternative 3' splice site 
Figure 3

\section{Multiple intronic repeats lead to distinct mature RNAs.}

(a) An individual intron forms RNA pairing which promotes canonical splicing to linear RNA generation. (b-c) Base pairs flanking the circularized exons results in back-splicing and generation of circRNAs.

Blue arrows: intronic repeat elements.

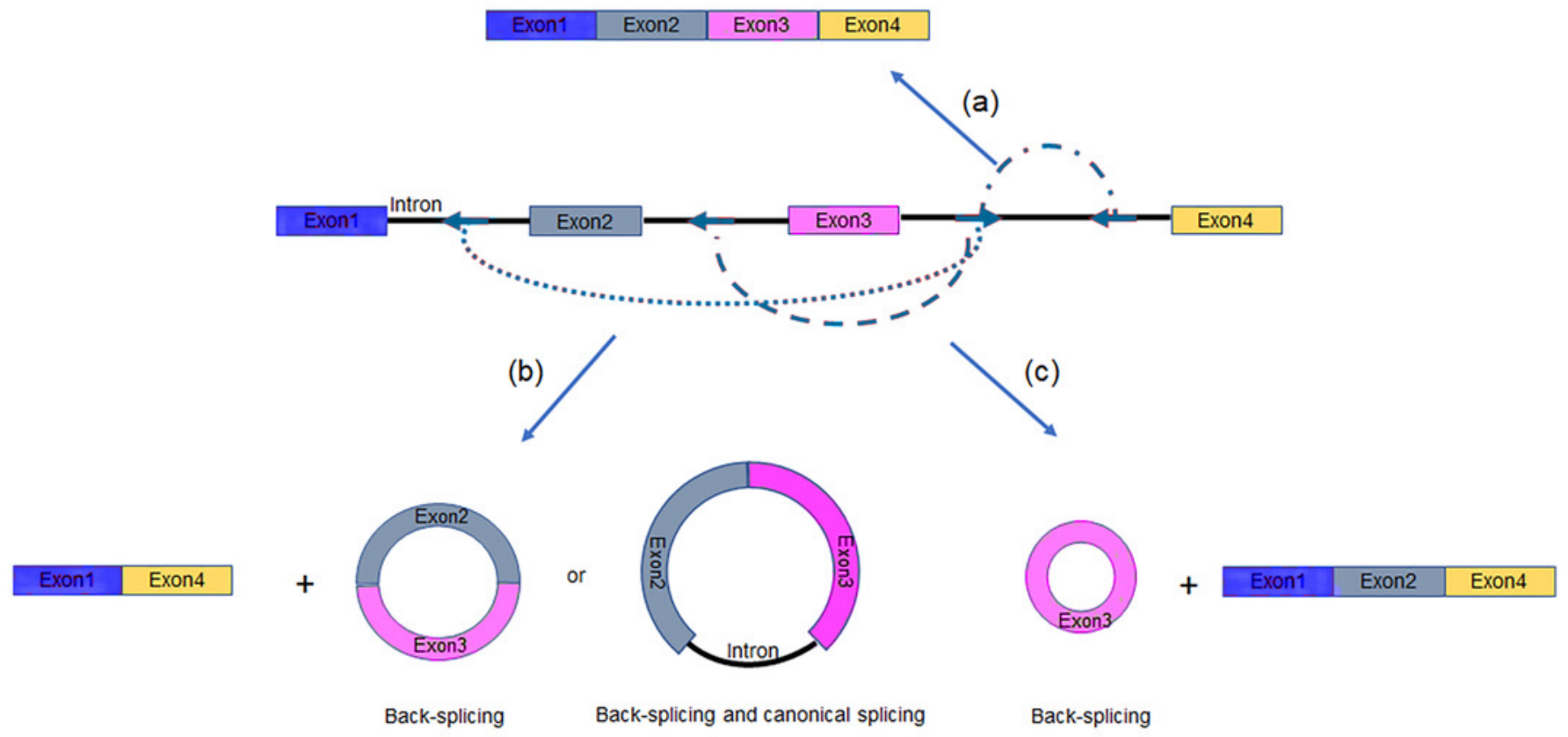




\section{Figure 4}

Methods used to enrich circRNAs.

(A)RPAD method (Panda et al., 2017). Linear RNAs are first depleted by RNase R digestion. After the remaining RNA is polyadenylated, a second round of depletion of poly(A)+ RNAs using oligo(dT) beads leaves a highly enriched population of circular RNAs. (B)Coupling A-Tailing with RNase R treatment in LiCl containing buffer (Xiao and Wilusz, 2019). Total RNA is treated with RNase R in KCl-containing buffer (left) . Total RNA is treated with an A-Tailing step followed by RNase R digestion in LiCl-containing buffer (right) .

Compared with the original image, the red/green colors were adjusted.

A
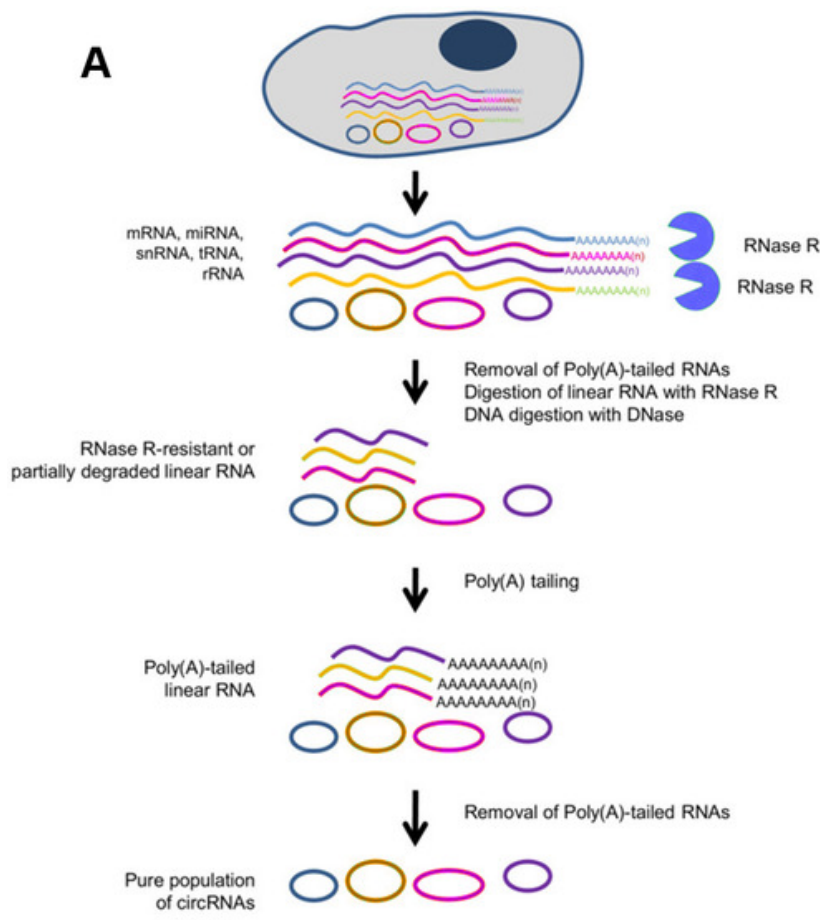

B

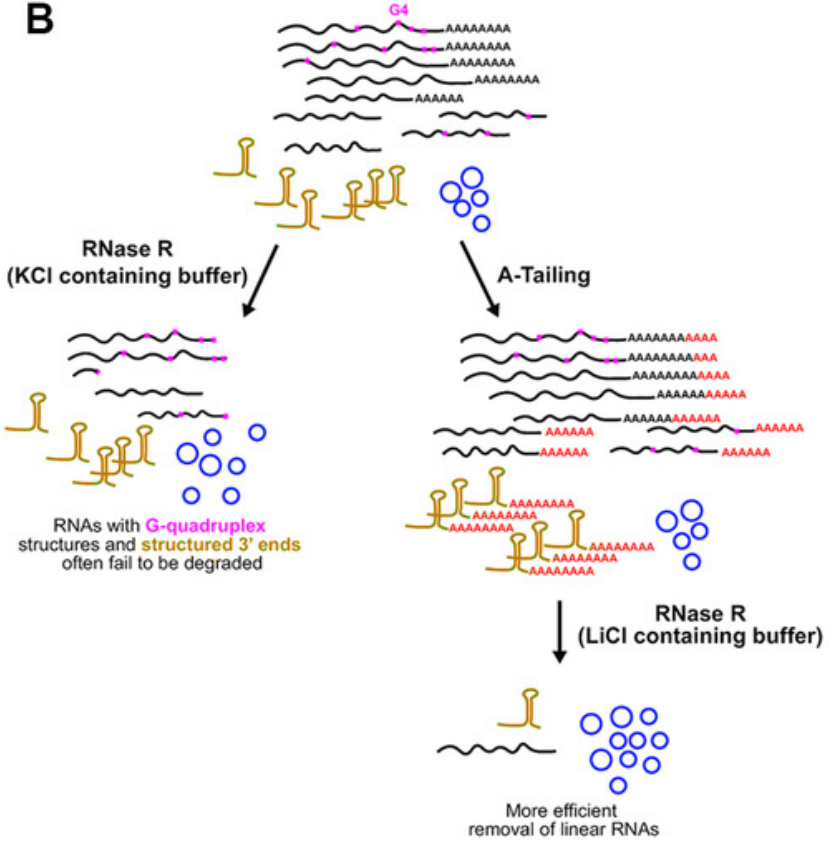




\section{Figure 5}

CircRNA-Rolling Circle Amplification (circRNA-RCA) method enables identification of circRNA variants (Das et al., 2019).

Firstly, RNase $\mathrm{R}$ is used to enrich the circRNA. Then, the enriched circRNA is reverse-transcribed with RNase $\mathrm{H}$-minus reverse transcriptase and cDNA is amplified by PCR, using full-length primers. Finally, the fulllength circRNA PCR products are sequenced by Sanger method to identify circRNA variants.

Black arrowheads: back-splice site. Compared with the original image, the red/green colors were adjusted. 
Enriched circRNAs

Full-length cDNA synthesis

Full-length circRNA PCR

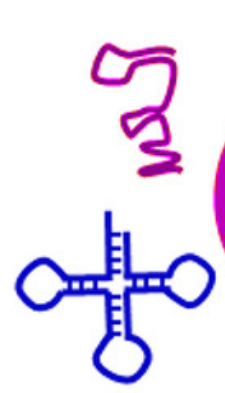

RNase R treatment

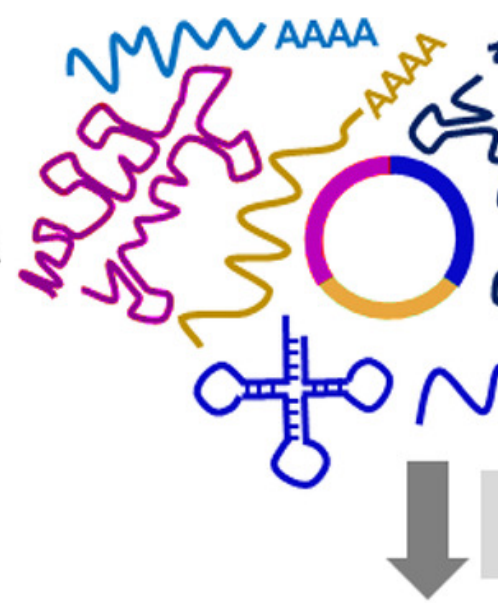

M

Total RNA

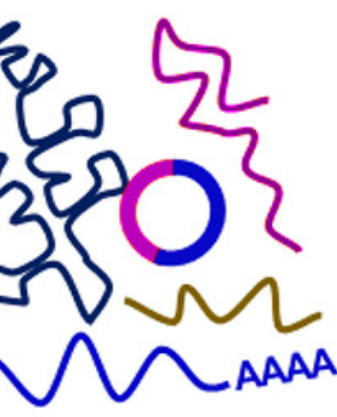

RNase H-minus RT
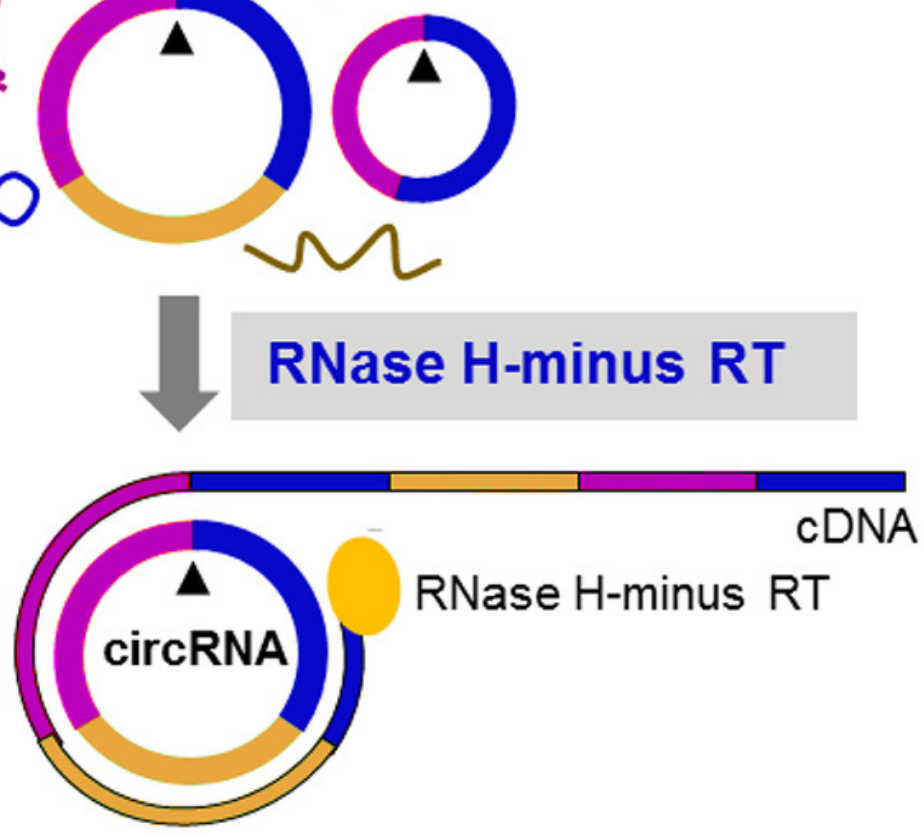

Full-length PCR

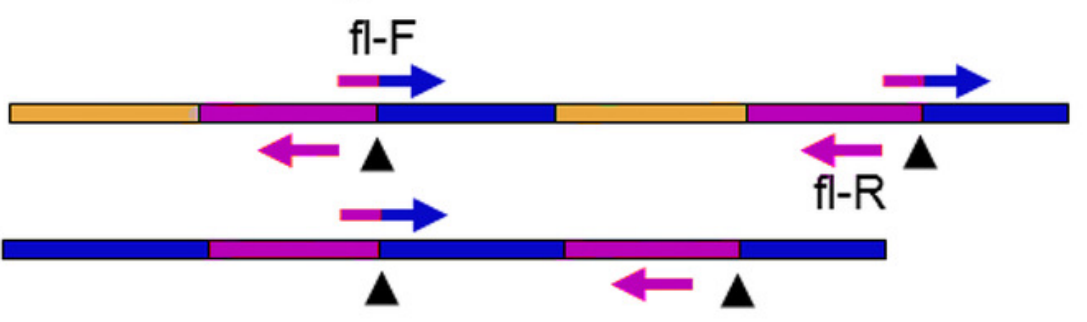

Sanger sequencing

Full-length circRNA sequences 


\section{Table $\mathbf{1}$ (on next page)}

Recently published bioinformatic tools used for AS detection in circRNAs 
Table 1:

Recently published bioinformatic tools used for AS detection in circRNAs

\begin{tabular}{|c|c|c|c|c|}
\hline Algorithm & Sample & $\begin{array}{l}\text { Sequencing } \\
\text { platform }\end{array}$ & $\begin{array}{l}\text { Application and } \\
\text { limitations }\end{array}$ & Reference \\
\hline CIRI-AS & $\begin{array}{l}\text { RNase } \mathrm{R} \text { - or RNase } \\
\mathrm{R}+\text { treated samples }\end{array}$ & $\begin{array}{l}\text { Illumina HiSeq } \\
2500\end{array}$ & $\begin{array}{l}\text { Detects AS within } \\
\text { circRNAs but has lower } \\
\text { sensitivity for short } \\
\text { reads }\end{array}$ & $\begin{array}{l}\text { Gao et al., } \\
2016\end{array}$ \\
\hline FUCHS & $\begin{array}{l}\text { rRNA- or rRNA- } \\
\text { /RNase R+ treated } \\
\text { samples) }\end{array}$ & $\begin{array}{l}\text { Illumina MiSeq } \\
\text { system or } \\
\text { Illumina } \\
\text { HiSeq } 2500\end{array}$ & $\begin{array}{l}\text { Identifies alternative } \\
\text { spliced circles and } \\
\text { visualizes the coverage } \\
\text { profile of circRNAs }\end{array}$ & $\begin{array}{l}\text { Metge et } \\
\text { al., } 2017\end{array}$ \\
\hline CIRCexplorer2 & $\begin{array}{l}\operatorname{Poly}(\mathrm{A})+, \operatorname{poly}(\mathrm{A})- \\
\text { and/or RNase R- } \\
\text { treated samples }\end{array}$ & $\begin{array}{l}\text { Illumina HiSeq } \\
2000\end{array}$ & $\begin{array}{l}\text { Detects alternative } \\
\text { (back-) splicing } \\
\text { circRNAs and parallel } \\
\text { poly(A)+ RNA-seq is } \\
\text { needed }\end{array}$ & $\begin{array}{l}\text { Zhang et } \\
\text { al., 2016a }\end{array}$ \\
\hline CircSplice & $\begin{array}{l}\text { rRNA-/RNase R- } \\
\text { treated samples }\end{array}$ & $\begin{array}{l}\text { Illumine Hiseq } \\
2500\end{array}$ & $\begin{array}{l}\text { AS detection between } \\
\text { cancer and normal } \\
\text { conditions }\end{array}$ & $\begin{array}{l}\text { Feng et al., } \\
2019\end{array}$ \\
\hline circseq_cup & $\begin{array}{l}\text { rRNA-/RNase R- } \\
\text { treated samples }\end{array}$ & $\begin{array}{l}\text { Illumina Hiseq } \\
3000\end{array}$ & $\begin{array}{l}\text { Assembly of full-length } \\
\text { circRNAs }\end{array}$ & $\begin{array}{l}\text { Ye et al., } \\
2017\end{array}$ \\
\hline CircAST & $\begin{array}{l}\text { rRNA-/RNase R- } \\
\text { treated samples }\end{array}$ & $\begin{array}{l}\text { Illumina Hiseq } \\
1500\end{array}$ & $\begin{array}{l}\text { Assembles and } \\
\text { quantifies exonic } \\
\text { circRNA isoforms but } \\
\text { may miss intronic or } \\
\text { intergenic circRNAs }\end{array}$ & $\begin{array}{l}\text { Wu et al., } \\
2019\end{array}$ \\
\hline CIRI-full & $\begin{array}{l}\text { rRNA-/RNase R- } \\
\text { treated samples } \\
\text { without } \\
\text { fragmentation }\end{array}$ & $\begin{array}{l}\text { Illumina HiSeq } \\
2500 \text { (PE250 or } \\
\text { PE300) }\end{array}$ & $\begin{array}{l}\text { Reconstructs and } \\
\text { quantifies circRNA } \\
\text { isoforms by utilizing } \\
\text { longer RNA-seq data }\end{array}$ & $\begin{array}{l}\text { Zheng et } \\
\text { al., } 2019\end{array}$ \\
\hline
\end{tabular}


Abbreviations:rRNA-: ribosomal RNA depletion; poly(A)+: polyadenylated RNA; poly(A)-: non-polyadenylated RNA; PE: paired-end. 